\title{
1 A 3D paper-based enzymatic fuel cell for self-powered, low- 2 cost glucose monitoring
}

3 Christopher Fischer ${ }^{1}$, Arwa Fraiwan ${ }^{2}$, and Seokheun Choi ${ }^{2 *}$

$4{ }^{1}$ Briarcliff High School, 444 Pleasantville Rd, Briarcliff Manor, NY, USA

$5 \quad{ }^{2}$ Bioelectronics \& Microsystems Laboratory, Department of Electrical \& Computer

6 Engineering, State University of New York at Binghamton, 4400 Vestal Pkwy,

7 Binghamton, NY, USA

$9 \quad{ }^{*}$ Corresponding author: Prof. Choi, Bioelectronics \& Microsystems Laboratory,

11 Binghamton, 4400 Vestal Pkwy, Binghamton, NY 13902, USA, E-mail:

sechoi@binghamton.edu

Abstract: In this work, we demonstrate a novel low-cost, self-powered paper-based biosensor for glucose monitoring. The device operating mechanism is based on a glucose/oxygen enzymatic fuel cell using an electrochemical energy conversion as a transducing element for glucose monitoring. The self-powered glucose biosensor features (i) a 3D origami paper-based structure for easy system integration onto paper, (ii) an air-cathode on paper for low-cost production and easy operation, and (iii) a screen printed chitosan/glucose oxidase anode for stable current generation as an analytical signal for glucose monitoring. The sensor showed a linear range of output current at 1 to $5 \mathrm{mM}$ glucose $\left(\mathrm{R}^{2}=0.996\right)$ with a sensitivity of $0.02 \mu \mathrm{A} \mathrm{mM}{ }^{-1}$. The advantages offered by such a device, including a low cost, lack of external power sources/sophisticated external transducers, and the capacity to rapidly generate reliable results, are well suited for the clinical and social settings of the developing world.

Keywords: Self-powered sensors, enzymatic fuel cells, glucose monitoring, biosensors, paper-based devices 


\section{Introduction}

2 Due to the stringent control of physiological blood glucose concentration being the only

3 way for diabetic patients to avoid life-threatening medical complications, glucose

4 monitoring is integral to diabetes management (Nathan, 2015). During the last several

5 decades, there have been significant advances in the field of glucose monitoring,

6 leading to highly improved blood glucose meters, continuous glucose monitoring

7 systems, and non-invasive monitoring technologies (Vashist, 2013). In developed

8 countries where healthcare infrastructure is well established, self-monitoring of blood

9 glucose levels has been found to be effective and economically accessible to the public

10 (Lawrence et al., 2014). However, in many resource-limited countries which are still

11 unable to cope with other fatal diseases due to the lack of laboratory infrastructure and

12 trained personnel, first priority is generally not given to diabetes care (Majikela-

13 Dlangamandla et al., 2006). Even widely used glucose meters and test strips are 14 extremely expensive in those low-income regions, given that a large proportion of the

15 population lives on less than $\$ 2$ per day (Collins et al., 2009). Although developing 16 countries have a high prevalence of diabetes and its complications, there is no

17 framework for diabetes monitoring and surveillance. Therefore, there is an urgent need

18 to develop a simple, low-cost, easily operable/accessible, disposable, and self-powered

19 glucose sensor that does not require sophisticated and expensive instrumentation,

20 allowing individuals in those countries to self-monitor their blood glucose at home.

Recently, paper-based microfluidic devices ( $\mu$ PADs) have received considerable

22 attention as an alternative technology for developing simple, low-cost, and disposable

23 platforms for glucose monitoring (Noiphung et al., 2013; Nie et al., 2010; Sechi et al., 
2013; Dungchai et al., 2009; Chun et al., 2014; Martinez et al., 2007). The application of $\mu$ PADs for glucose monitoring presents many advantages over current techniques; (i) redox enzymes for glucose oxidation can be readily immobilized in the paper matrix via physical adsorption without active pumps through the wicking power of hydrophilic paper, (ii) many microfluidic components can be integrated in paper with intricate 3D microfluidic channels to create a fully functional glucose sensor for superior analytical performance, (iii) a wide range of electrode materials can be easily screen-printed on the paper without expensive cleanroom facilities, and (iv) the application of origami for the overall $\mu$ PADs design will enable a simple one-step assembly process.

Several $\mu$ PAD-based colorimetric sensors have been proposed for simple and lowcost glucose monitoring (Martinez et al., 2007; Sechi et al., 2013; Chun et al., 2014). Based on enzymatic oxidation, in which a color change is associated with the presence of glucose, the colorimetric assay enables the naked eye to monitor the color intensity according to the glucose concentration. This monitoring approach, however, is extremely subjective, providing only "yes/or" or semi-quantitative analysis (Lee and Choi, 2015; Choi, 2015). High sensitivity, accuracy, and precision can be achieved with other detection methods such as fluorescence, chemiluminescence, and electrochemistry. In particular, an electrochemical detection method for $\mu$ PADs has been considered as an attractive alternative platform to conventional amperometric glucose monitoring due to its high sensitivity and selectivity (Li et al., 2015; Dungchai et al., 2009; Nie et al., 2010) . The electrochemical assay determines glucose concentration by measuring changes in current from the enzymatic oxidation. However, even $\mu$ PAD-based electrochemical sensors might not be suitable for use in resource-limited settings due to requiring (i) 
1 sophisticated external instrumentation for the quantitative readouts and (ii) sufficient

2 electrical energy for powering the technique. Furthermore, the electrochemical

3 measurements require additional electrode integration for the reference to control

4 studies and prevent unwanted system perturbations, increasing device costs and

5 fabrication complexity.

6 Recently, a novel concept for glucose sensors using electrochemical energy

7 transformation in an enzymatic fuel cell (EFC) as a transducing technique has received

8 significant interest (Katz et al., 2001; Liu et al., 2012; Arechederra and Minteer, 2011).

9 Their electron harvesting/storing capabilities from enzymes contributed to the

10 development of self-powered glucose sensors, in which glucose is oxidized at the

11 anode while oxygen is reduced at the cathode and a potential difference between the

12 electrodes is developed. Thus, the electrical output of the EFC as an analytical signal is

13 proportional to the glucose concentration. The unique features of EFC-based

14 biosensors, such as not having the need for external power sources or sophisticated

15 external transducers and simple sensing mechanisms, make them promising sensor

16 platforms for applications in resource-limited countries not only for the glucose

17 monitoring but also for the analysis of various other chemical/biological agents, such as

18 mercuric ions (Wen et al., 2011), ascorbic acid (Zloczewska et al., 2014), acetaldehyde

19 (Zhang et al., 2012), ethylenediaminetetraacetic acid (EDTA) (Meredith and Minteer,

20 2011), and other human biomarkers (Wang et al., 2014; Gai et al., 2015).

21 In this work, we report the first demonstration of a 3D paper-based EFC for self22 powered glucose monitoring, which is extremely low cost $(\sim 0.15)$ and requires only a 
1 simple, inexpensive digital multi-meter $(\sim 2)$ for signal readout. The sensor operates

2 with a single drop $(20 \mu \mathrm{L})$ of glucose-including sample driven by capillary force through

3 patterned microfluidic pathways on paper. An air-breathing cathode on paper replaced

4 the common employed enzymatic cathode, providing a lower cost and higher practicality

5 in developing countries. Currently, reported work about a fully paper-based EFC

6 glucose sensor is either unavailable or quite limited. This proof-of-concept device will

7 enable the development of new types of self-powered biosensing assays in convenient, 8 easy-to-use paper packages.

\section{Materials and Methods}

\subsection{Operating Principle}

12 The operating principle of our paper-based EFC-based glucose sensor is described in

13 the schematic diagram of Fig. 1. When a sample containing glucose is introduced into 14 the paper reservoir, glucose oxidization is catalyzed by the glucose oxidase $\left(\mathrm{GO}_{\mathrm{x}}\right.$, a redox enzyme) immobilized at the anode. The enzyme then transfers its electrons to the

16 anodic surface through a direct electron transfer (DET) reaction. These electrons flow to

17 the air-cathode through the external circuit. Protons released by the oxidation reaction

18 travel through the paper reservoir toward the air-cathode. By using freely-available oxygen as the electron acceptor, the cathode completes the redox reaction. The open

20 circuit voltage between the electrodes and current/power generation from the EFC are a 21 function of glucose concentration in the sample, creating a self-powered glucose 22 monitoring system. 


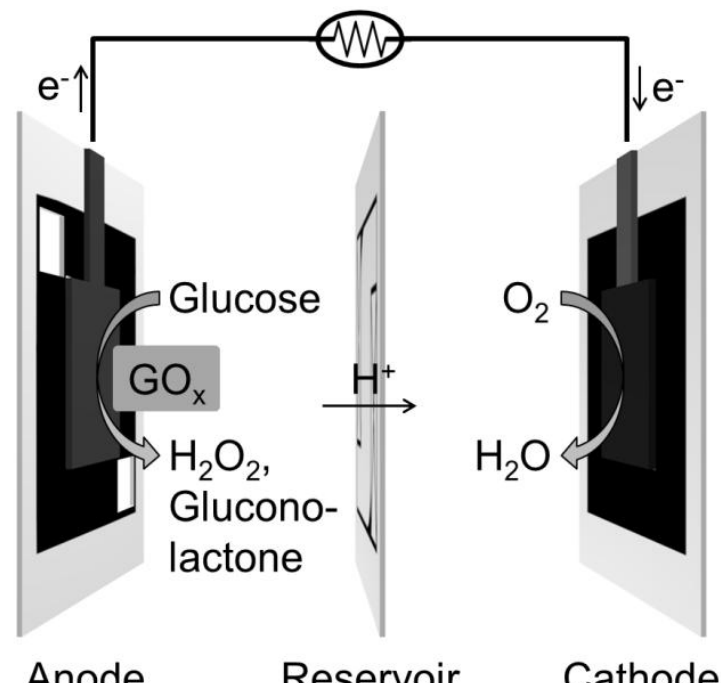

Fig. 1. Schematic of operation of a EFC-based glucose sensor: at the GOx anode, glucose is oxidized to gluconolactone/H2O2 and the released electrons are transferred to the anode surface and then through the external circuit. At the cathode, electrons and protons reduce 02 to water on the air-cathode.

$2 \quad 2.2$ Reagents

$3 \mathrm{GO}_{\mathrm{x}}$ from Aspergillus niger (CAS 9001-37-0), chitosan (CAS 9012-76-4), acetic acid

$4 \quad$ (CAS 64-19-7), Nafion ${ }^{\circledR}$ solution (CAS 31175-20-9), and D-glucose (CAS 50-99-7) were

5 purchased from Sigma-Aldrich. $0.10 \mathrm{M} \mathrm{pH} 7.0$ phosphate buffer solution (PBS) was

6 used as the supporting electrolyte. Activated carbon and graphite ink were purchased

7 from Cabot Corporation and Fisher Scientific, respectively. We also used a nickel

8 conductive spray purchased from MG Chemicals for the air-cathode. 


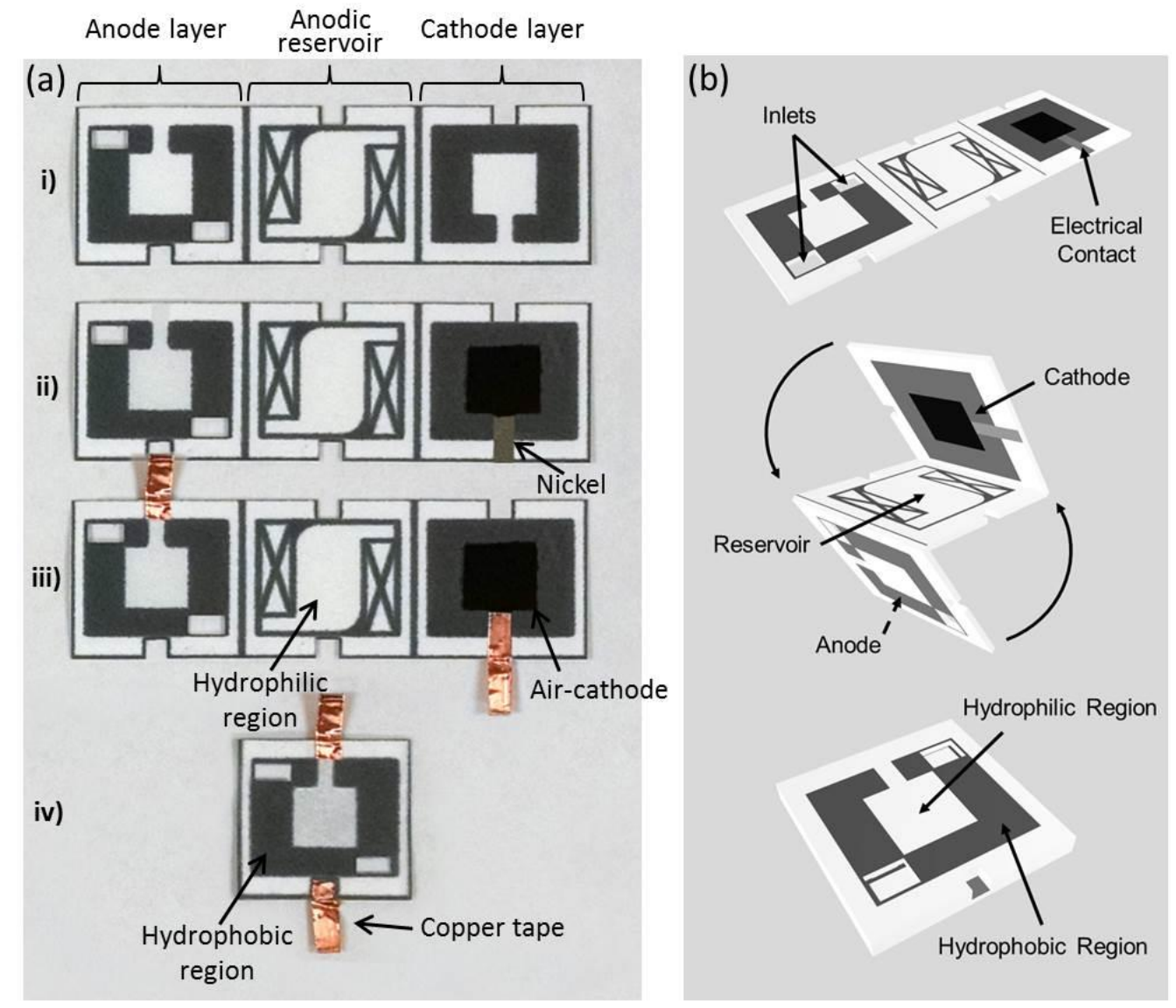

Fig. 2. (a) Intermediate steps for fabrication process of single cell EFC: step (i): wax-boundaries are formed; step (ii): electrodes are printed; step (iii) copper tape is pressed on; step (iv) cell is folded, glued, and flipped to expose the reservoir through the inlet holes. (b) Schematic diagrams of the origami EFC during assembly. The 3D EFC structure can be easily assembled from 2D sheets by simply folding along pre-defined creases and gluing.

\subsection{Device Fabrication}

\subsubsection{Wax-Boundaries and Anode Reservoir}

4 The paper-based EFC sensor was fabricated on a single paper sheet (Whatman \#1

5 filter paper) patterned into hydrophilic regions defined by printed wax boundaries with

6 controlled geometry using a commercially available solid-wax printer (XeroxColorQube

$78570 \mathrm{dn}$ ) (Fig. 2). The wax patterns were then heat treated at $150^{\circ} \mathrm{C}$ for 2 min to allow

8 for complete penetration of the wax through the paper. AutoCAD software was used to 
1 design the wax patterns on the paper sheet. The device consisted of three distinctive

2 regions (Fig. 2a); (i) the anode layer, (ii) the anode reservoir, and (iii) the air-cathode

3 layer, which were folded over each other to create one cell (Fig. 2b). The anode

4 reservoir, which holds the glucose sample to allow for prolonged electricity generation,

5 was employed not only for rapid sample absorption and promotion of immediate power

6 generation but also for selective proton transfer towards the cathode.

8 2.3.2 Fabrication of Bioanode and Air-Cathode

9 The anode was prepared on the back side of the left part of paper sheet (Fig. 2a) by

10 screen-printing graphite ink through a stencil onto the hydrophilic area. After drying, a

$1132 \mu \mathrm{L}$ aliquot of $\mathrm{GO}_{x}$ solution was uniformly spread over the anode. $\mathrm{GO}_{x}$ solution was

12 prepared by stirring $5 \mathrm{mg} \mathrm{GO}$ x dissolved in $0.5 \mathrm{~mL} 0.1 \mathrm{M} \mathrm{pH} 7.0$ PBS with $1 \mathrm{~mL}$ of $1 \%$

13 chitosan solution prepared in $1 \%$ acetic acid for 1 hour using a magnetic stirrer (Liu et

14 al., 2005). $\mathrm{GO}_{x}$ was used in this study due to its extremely specific catalytic activity

15 towards glucose, suggesting that contamination with other carbohydrates or alcohols in

16 any potential sample would not interfere with the biosensor's accuracy. The enzyme

17 was immobilized in chitosan, a biocompatible substance with a high specific surface 18 area, to facilitate the transfer of electrons and lead to better stability and higher

19 sensitivity of glucose (Chen et al., 2003; Kang et al., 2009). The bioanode was dried in

20 air overnight at $4^{\circ} \mathrm{C}$. Two inlets were cut on the anodic layer, which lead directly to the

21 anode reservoir, to allow for the introduction of anolyte. Capillary force then spread this

22 sample over the surface of the anode, filing the reservoir and allowing the start of the 23 redox reaction between $\mathrm{GO}_{x}$ and glucose. 
An activated carbon-based air-cathode was fabricated on the front side of the right part of the paper sheet (Fig. 2a). Our group previously constructed the air-cathode on

3 paper successfully by layering deposited Ni with activated carbon catalyst $\left(15 \mathrm{mg} / \mathrm{cm}^{2}\right)$

4 in a binder solution (Lee and Choi, 2015). The binder solution was prepared by mixing

$5150 \mu \mathrm{L}$ of DI water, $1200 \mu \mathrm{L}$ of $5 \mathrm{wt} \%$ Nafion solution, and $600 \mu \mathrm{L}$ of isopropanol. This air-

6 cathode (i) reduces cost over an order of magnitude compared to the conventional

7 biocathode ( $\$ 0.06$ vs. $\$ 3.10$ for the enzymatic biocathode utilizing Bilirubin Oxidase,

$8 \mathrm{BO}_{\mathrm{x}}$ ), (ii) increases stability in open air and at room temperature with the removal of a

9 highly fragile enzyme, and (iii) greatly simplifies the fabrication process. The wax-

10 patterned paper with electrodes was subsequently cut with a computer-controlled laser

11 cutting and engraving system (Universal Laser System, VLS 3.5), and copper tape was

12 used as contact pads to facilitate connection to the measurement apparatus.

13 The 3D EFC-based sensor was assembled from the 2D sheet by simply folding 14 along pre-defined creases and gluing with adhesive spray. The overall size of the folded 15 EFC was $2.5 \mathrm{~cm} \times 2.5 \mathrm{~cm}$ with an anodic surface area of $1 \mathrm{~cm} \times 1 \mathrm{~cm}$.

16 The total material cost of the fabricated device was reduced significantly from

17 previously reported EFCs to about $\$ 0.15$ for a single device, an order of magnitude less 18 expensive than a single OneTouch $\AA$ test strip (Filter paper: $\$ 0.02, \mathrm{GO}_{\mathrm{x}}: \$ 0.06$, Nafion 19 Solution: $\$ 0.05$; Spray adhesive: $\$ 0.01$; Nickel spray: $\$ 0.01)$.

\subsection{Measurement Setup}

22 We measured the potential between the anode and the cathode with a data acquisition 23 system (NI, USB-6212) and recorded the results every 6 seconds via a customized 
1 LabVIEW interface. An external resistor was used to close the circuit. The current was

2 calculated via Ohm's law, and the power output was calculated via P $=$ V X I.

\section{Results and Discussion}

\subsection{Sample Introduction}

6 Once the device was fully folded and assembled, two inlets offered access to the anode reservoir through which the glucose solution was introduced into the EFC. The solutions

8 wicked through the paper substrate and across the surface of the bioanode without the 9 need for any external pumps. This adsorption promoted glucose attachment to the 10 anode and immediate electron transfer from $\mathrm{GO}_{x}$, resulting in a short start-up time. The

11 large pore sized filter paper (Whatman \#1; 10 $\mu \mathrm{m}$ ) allowed a higher volume of sample

12 to be absorbed into the reservoir and consequently a longer duration of stable current 13 generation. To maximize the cathodic reactions, once the sample was added into the 14 reservoir, the device was flipped over to expose air-cathodes to the air.

\subsection{Open circuit voltages}

17 The open circuit voltages (OCVs) from EFC sensors were first measured. The OCV with

18 a $5 \mathrm{mM}$ glucose sample gradually increased and reached a value of approximately 240 $\mathrm{mV}$. All experiments were repeated five times and the results showed variation of less

20 than $\pm 2 \%$. This corroborates the repeatability of our proposed glucose monitoring

21 scheme. Previous self-powered EFC sensors used the OCV outputs to measure the

22 concentration of glucose in the anolyte, as OCVs increased logarithmically with the 
1 increase of sample concentration. In this work, however, differences in current 2 generation of the EFCs were utilized to quantify the different detected glucose 3 concentrations.
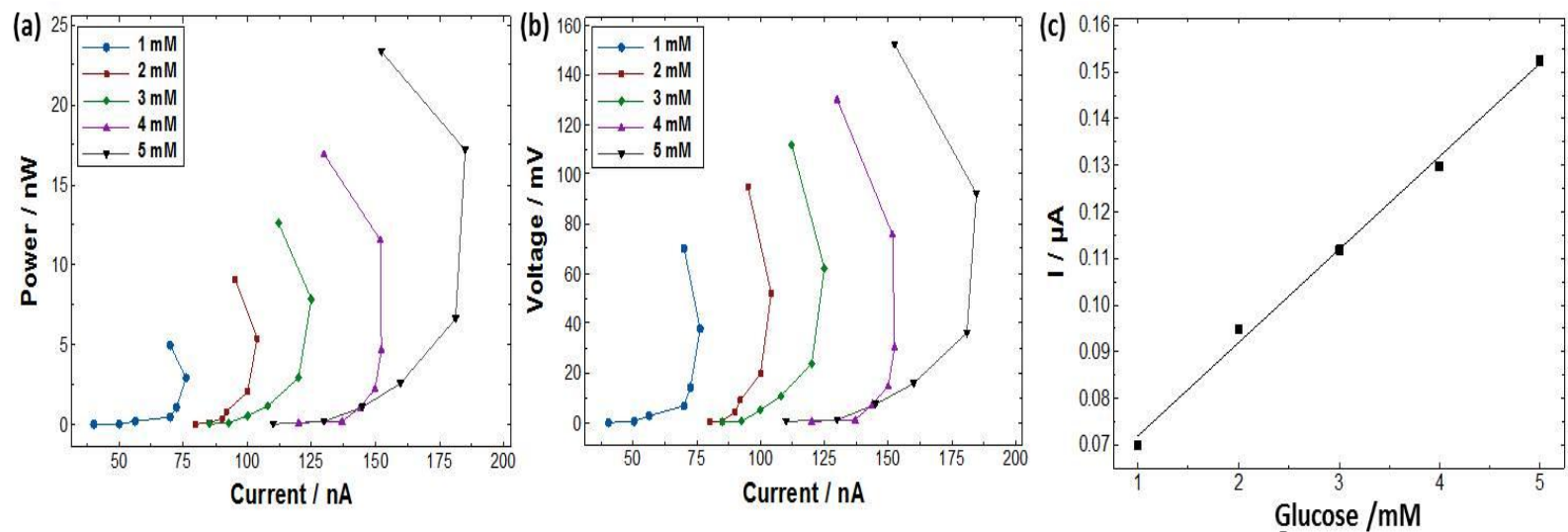

Fig. 3. Power output (a) and polarization curves (b) showing levels of power generation with varying concentrations of glucose. (c) Calibration curve of glucose biosensor's output current vs. the concentration of glucose in $\mathrm{mM}$ with a $1 \mathrm{M} \Omega$ resistor connecting the device's electrical leads.

\subsection{Glucose monitoring}

Fig. 3 shows power outputs (a) and polarization curves (b) of the device with varying concentrations of glucose $(1 \mathrm{mM}, 2 \mathrm{mM}, 3 \mathrm{mM}, 4 \mathrm{mM}$, and $5 \mathrm{mM})$. The curves were derived and calculated based on the stabilized current value at a given external resistance $(1 \mathrm{M} \Omega, 500 \mathrm{k} \Omega, 200 \mathrm{k} \Omega, 100 \mathrm{k} \Omega, 50 \mathrm{k} \Omega, 10 \mathrm{k} \Omega$, and $1 \mathrm{k} \Omega)$. The maximum power outputs were obtained under a $1 \mathrm{M} \Omega$ resistor while the maximum currents were

11 observed for $500 \mathrm{k} \Omega$ resistor for all five glucose concentrations. This clearly shows that our devices have a high internal resistance. The internal resistance of the EFC sensor was approximately $1 \mathrm{M} \Omega$, which is several orders of magnitude higher than the values

14 previously reported in the literature (Minteer et al., 2007; Minteer et al., 2012). The high

15 internal resistance observed in our experiments is most likely caused by the poor 
1 electron transfer from the enzymes to the anode surface, as well as the inefficient

2 interaction between the enzyme and the anode. To date, substantial research efforts

3 have been devoted to decrease the internal resistance of EFCs mainly by using 3D

4 micro-/nano-structured anodes, increasing loading or specific activity of the enzymes,

5 and adding mediators to facilitate the electron transfer (Arechederra and Minteer, 2011;

6 Cosnier et al., 2014; Yu and Scott, 2010). However, none of these approaches has

7 been applied to paper-based platforms. Our future work will be veered towards testing

8 the applicability of the aforementioned techniques to paper-based devices.

Fig. 3 shows rapid decline of the voltage/power at higher current under low load resistor, suggesting that the performance of the EFC is quite limited by mass transport 11 loss. Therefore, it is also important to enlarge the anodic reservoir to accommodate for 12 a sufficient supply of glucose sample to the anodic enzymes at rates at least equivalent 13 to the current generation rate. Although the paper-based EFC developed in this work 14 was not able to generate high current or power, it has a remarkable potential for use as 15 a self-powered glucose sensor, as it generated enough current/power to sensitively resolve varying glucose concentrations Fig. $3 c$ shows the biosensor's calibration curve

17 with a $1 \mathrm{M} \Omega$ resistor. A high linearity is shown $\left(R^{2}=0.996\right)$ with a sensitivity of $0.02 \mu \mathrm{A}$ $18 \mathrm{mM}^{-1}$.

20 before operating them, the measurements made with these devices show good

21 agreement with the previous results. At this point we continue to store the devices and 22 test them to determine their shelf-life. 


\section{Conclusions}

2 We report for the first time a 3D paper-based self-powered EFC sensor that allows for

3 the low-cost detection of glucose in biological solutions. This device used a screen

4 printed chitosan/GO $\mathrm{GO}_{\mathrm{x}}$ anode to promote steady current generation and a paper-based

5 air-cathode to increase stability in open air while significantly decreasing the cost. The

6 proposed EFC-based sensor has numerous advantages over previous developments:

7 (i) the use of paper significantly decreases startup time and allows it to function with a

8 sample volume as small as $20 \mu \mathrm{L}$, (ii) this sensing platform can be easily integrated with

9 other various enzymatic assays, (iii) the device is safe, environmentally friendly, and

10 disposable via incineration; and (iv) it is the most inexpensive glucose detecting system

11 to date. Importantly, the EFC-based self-powered sensor will require minimal external

12 instrumentation for obtaining quantitative signal outputs.

13

14 Acknowledgement

15 This work is supported by NSF (ECCS \#1503462).

17 REFERENCES

18 Arechederra, R.L., Minteer, S.D., 2011. Anal Bioanal Chem 400, 1605-1611.

19 Chen, X., Jia, J., Dong, S., 2003. Electroanalysis 15, 608-612.

20 Choi, S., 2015. Biosensors and Bioelectronics 69, 8-25. 
1 Chun, H.J., Park, Y.M., Han, Y.D., Jang, Y.H., Yoon, H.C., 2014. Biochip J 8, 218-226.

2 Collins, D., Morduch, J., Rutherford, S., Ruthven, O., 2009. Portfolios of the Poor: How

3 the World's Poor Live on $\$ 2$ a Day, Princeton University Press.

4 Cosnier, S., Holzinger, M., Goff, A.L., 2014. Front Bioeng Biotechnol 2, 45.

5 Dungchai, W., Chailapakul, O., Henry, C.S., 2009. Anal Chem 81. 5821-5826.

6 Gai, P., Ji, Y., Wang., W., Song, R., Zhu, C., Chen, Y., Zhang, J., Zhu, J., 2015. Nano

7 Energy, In print (doi:10.1016/j.nanoen.2015.03.035)

8 Katz, E., Bückmann, A.F., Willner, I., 2001. J Am Chem Soc 123, 10752-70753.

9 Kang, X., Wang, J., Wu, H., Aksay, I.A., Liu, J., Lin, Y., 2009. Biosensors and 10 Bioelectronics 25, 901-905.

11 Lawrence C.S.K., Tan S.N., Floresca, C.Z., 2014. Sensors and Actuators B 193, 53612541.

13 Lee, H., Choi, S., 2015. Lab Chip 15, 391-398.

14 Li, X., Zhao, C., Liu, X., 2015. Microsystems \& Nanoengineering 1, 15014.

15 Liu, Y., Wang, M., Zhao, F., Xu, Z., Dong, S., 2005. Biosensors and Bioelectronics 21, $16 \quad 984-988$.

17 Liu, Z., Cho, B., Ouyang, T., Feldman, B., 2012. Anal Chem 84, 3403-3409.

18 Majikela-Dlangamandla, B., Isiavwe, A., Levitt, N., 2006. Diabetes Voice 51, 28-31 
1 Martinez, A.W., Phillips, S.T., Butte, M.J., Whitesides, G.M., 2007. Angew Chem Int Ed $246,1318-1320$.

3 Meredith, M.T., Minteer, S.D., 2011. Anal Chem 83, 5436-5441.

4 Minteer S.D., Atanassov, P., Luckarift, H.R., Johnson, G.R., 2012. Materials Today 15, $5 \quad 166-173$.

6 Minteer, S.D., Liaw, B.Y., Cooney, M.J., 2007. Current Opinion in Biotechnology 18, $7 \quad 228-234$.

$8 \quad$ Nathan, D.M., 2015. JAMA 314, 1052-1062.

9 Nie, Z., Nijhuis, C.A., Gong, J., Chen, X., Kumachew, A., Martinez, A.W., Narovlyansky, 10 M., Whitesides, G.M., 2010. Lab Chip 10, 477-483.

11 Noiphung, J., Songjaroen, T., Dungchai, W., Henry, C.S., Chailapakul, O., 12 Laiwattanapaisal, W., 2013. Analytica Chimica Acta 788, 39-45.

13 Sechi, D., Greer, B., Johnson, J., Hashemi, N., 2013. Anal Chem 85, 10733-10737.

14 Vashist, S.K., 2013. Diagnostics 3, 385-412.

15 Wang, Y., Ge, L., Wang, P., Yan, M., Yu, J., Ge, S., 2014. Chem Commun 50, 1947161949.

17 Wen, D., Deng, L., Guo, S., Dong, S., 2011. Anal Chem 83, 3968-3972.

18 Yu, E.H., Scott, K., 2010. Energies 3, 23-42. 
1 Zloczewska, A., Celebanska, A., Szot, K., Tomaszewska, D., Opallo, M., Jönsson-

2 Niedziolka, M., 2014. Biosensors and Bioelectronics 54, 455-461.

3 Zhang, L., Zhou, M., Dong, S., 2012. Anal Chem 84, 10345-10349.

4 Figure Captions

5 Figure 1. Schematic of operation of a EFC-based glucose sensor: at the GOx anode, glucose is 6 oxidized to gluconolactone/ $\mathrm{H} 2 \mathrm{O} 2$ and the released electrons are transferred to the anode surface 7 and then through the external circuit. At the cathode, electrons and protons reduce 02 to water on 8 the air-cathode.

Figure 2. (a) Intermediate steps for fabrication process of single cell EFC: step (i): wax-boundaries are formed; step (ii): electrodes are printed; step (iii) copper tape is pressed on; step (iv) cell is folded, glued, and flipped to expose the reservoir through the inlet holes. (b) Schematic diagrams of the origami EFC during assembly. The 3D EFC structure can be easily assembled from 2D sheets by simply folding along pre-defined creases and gluing.

Figure 3. Power output (a) and polarization curves (b) showing levels of power generation with varying concentrations of glucose. (c) Calibration curve of glucose biosensor's output current vs. the concentration of glucose in $\mathrm{mM}$ with a $1 \mathrm{M} \Omega$ resistor connecting the device's electrical leads. 